\title{
MicroRNA-455-3p promotes TGF- $\beta$ signaling and inhibits osteoarthritis development by directly targeting PAK2
}

\author{
Shu Hu', Xiaoyi Zhao', Guping Mao', Ziji Zhang ${ }^{1}$, Xingzhao Wen'1 Chengyun Zhang ${ }^{1}$, Weiming Liao ${ }^{1}$ and
} Zhiqi Zhang ${ }^{1}$

\begin{abstract}
MicroRNAs (miRNAs, miR) play a key role in the pathogenesis of osteoarthritis (OA). Few studies have examined the regulatory role of P21-activated kinases (PAKs), a family of serine/threonine kinases, in OA. The aim of this study was to determine whether miR-455-3p can regulate cartilage degeneration in OA by targeting PAK2. MiR-455-3p knockout mice showed significant degeneration of the knee cartilage. MiR-455-3p expression increased and PAK2 expression decreased in the late stage of human adipose-derived stem cell (hADSC) chondrogenesis and in chondrocytes affected by OA. Furthermore, in both miR-455-3p-overexpressing chondrocytes and PAK2-suppressing chondrocytes, cartilage-specific genes were upregulated, and hypertrophy-related genes were downregulated. A luciferase reporter assay confirmed that miR-455-3p regulates PAK2 expression by directly targeting the $3^{\prime}$-untranslated regions (3'UTRs) of PAK2 mRNA. IPA-3, a PAK inhibitor, inhibited cartilage degeneration due to OA. Moreover, suppressing PAK2 promoted R-Smad activation in the TGF/Smad signaling pathway in chondrocytes. Altogether, our results suggest that miR-455-3p promotes TGF- $\beta / S$ mad signaling in chondrocytes and inhibits cartilage degeneration by directly suppressing PAK2. These results thus indicate that miR-455-3p and PAK2 are novel potential therapeutic agents and targets, respectively, for the treatment of OA.
\end{abstract}

\section{Introduction}

Osteoarthritis $(\mathrm{OA})$, characterized by osteophyte formation and articular cartilage degradation, is the leading cause of chronic disability in older adults ${ }^{1}$. However, the underlying pathogenesis is still unknown ${ }^{2}$. In recent years, microRNAs (miRNAs, miR) have attracted considerable attention due to their critical role in the regulation of gene expression through binding to the $3^{\prime}$-untranslated regions ( $3^{\prime}$ UTRs) of target genes ${ }^{3}$. For example, miR-21 regulates the development of OA by targeting GDF- $5^{4}$, whereas miR-139 induces apoptosis in OA chondrocytes by targeting MCPIP $1^{5}$. We previously reported the upregulation

Correspondence: Weiming Liao (liaowmsysu@163.com) or Zhiqi Zhang (zhzhiqi@mail.sysu.edu.cn)

'Department of Joint Surgery, the First Affiliated Hospital of Sun Yat-sen University, Guangzhou 510080, China

These authors contributed equally: Shu Hu, Xiaoyi Zhao, Guping Mao of miR-455-3p during early chondrogenesis of human adipose-derived stem cells (hADSCs) ${ }^{6}$ and ATDC5 cells $^{7,8}$. Subsequently, we hypothesized that miR-455-3p may play a key role in chondrogenesis or cartilage degeneration.

The TGF- $\beta /$ Smad signaling pathway is an important regulator of cartilage anabolism. This pathway can enhance the synthesis of type II collagen and aggrecan, and prevent cartilage degradation ${ }^{9,10}$. TGF- $\beta /$ Smad signaling is initiated by ligand binding to the type II receptor, which subsequently phosphorylates the type I receptors, both of which are serine/threonine kinase receptors. For canonical pathways, the activated receptor complex phosphorylates receptor-regulated Smad proteins (RSmad: Smad2/3). The phosphorylated R-Smad then associates with Smad4 to form a complex that

\section{(c) The Author(s) 2019}

(c) (i) Open Access This article is licensed under a Creative Commons Attribution 4.0 International License, which permits use, sharing, adaptation, distribution and reproduction BY in any medium or format, as long as you give appropriate credit to the original author(s) and the source, provide a link to the Creative Commons license, and indicate if changes were made. The images or other third party material in this article are included in the article's Creative Commons license, unless indicated otherwise in a credit line to the material. If material is not included in the article's Creative Commons license and your intended use is not permitted by statutory regulation or exceeds the permitted use, you will need to obtain permission directly from the copyright holder. To view a copy of this license, visit http://creativecommons.org/licenses/by/4.0/. 
translocates into the nucleus, where it interacts with transcription factors to activate distinct subsets of genes.

P21-activated kinases (PAKs) are a family of serine/ threonine kinases that are classified into two groups: groups I (PAK1-3) and II (PAK4-6). PAKs contain an autoinhibitory domain, which can be activated by the small GTP-binding proteins $\mathrm{Cdc} 42$ and Rac $1^{11,12}$. PAKs have multiple biological functions ${ }^{13}$, and PAK2 is widely distributed throughout the body. Several substrates of PAK2, such as c-Jun, and cellular events associated with PAK2, including tumorigenesis, have been identified ${ }^{14,15}$. PAK2 also helps mediate COX2 expression in papillomas through the NF- $\mathrm{kB}$ pathway activation $^{16}$. Moreover, PAK2 inhibits the TGF- $\beta$ signaling pathway by interfering with the R-Smad interaction in Madin-Darby canine kidney (MDCK) epithelial cells ${ }^{17}$. However, no studies have assessed the effects of PAK2 on OA chondrocytes. Using miRNA target-prediction algorithms, we found that miR-455-3p potentially regulates PAK2 expression. Based on this information and the regulatory role of PAK2 in the TGF- $\beta$ signaling pathway, we hypothesized that miR-455-3p may regulate the metabolism of chondrocytes by modulating PAK2 expression.

In this study, we report the role of PAK2 in OA chondrocytes and demonstrate that miR-455-3p promotes TGF- $\beta$ signaling and inhibits cartilage degeneration in OA chondrocytes by directly targeting PAK2.

\section{Materials and methods}

This study adhered to the standards of the Ethics Committee on Human Experimentation of The First Affiliated Hospital at Sun Yat-Sen University, China (IRB:2011011) and the Helsinki Declaration (2000). All participants provided informed consent.

\section{MiR-455-3p global knockout (KO) mouse model}

All mouse breeding and animal procedures were approved by the Animal Research Committee of The First Affiliated Hospital of Sun Yat-sen University, China (IRB: 2014C-028). MiR-455-3p global KO mice were generated using a transcription activator-like effector nuclease (TALEN) system, which was described in our previous study $^{18}$. Three pairs of KO and wild-type (WT) C57BL/6 mice were euthanized at either 5 months or 12 months of age, and their knee joints were collected. The knee joint sections were stained via immunohistochemistry and in situ hybridization for further analysis. After staining with Safranin O and Fast Green, the tissues were scored for histopathology by the modified Mankin scoring system ${ }^{19}$. The OA histopathology was quantified by summing the scores for the following four criteria: surface fissuring (0-3), pericellular matrix staining $(0-2)$, spatial arrangement of chondrocytes (0-3), and interterritorial matrix staining $(0-3)$. A higher score indicates more advanced OA histopathology.

\section{Cell isolation and culture}

OA cartilage samples were obtained from the knee joint of patients with $\mathrm{OA}$ who underwent total knee replacement surgery $(n=6$, mean \pm standard deviation [SD] age: $62.83 \pm 1.17$ years, male: 1 , female: 5), and control cartilage samples were obtained from patients with no history of OA or rheumatoid arthritis who underwent low limb amputation surgery due to sarcomas not involving the knee or ankle joints $(n=7$, mean \pm SD age: $19.29 \pm 5.94$ years, male: 4 , female: 3 ). The isolation method of primary human chondrocytes (PHCs) from cartilage was described previously ${ }^{20}$. PHCs were cultured in Dulbecco's modified Eagle's medium F12 (DMEM/F-12; Gibco Life Technology) supplemented with $5 \%$ fetal bovine serum (FBS; Gibco Life Technology) and 1\% penicillin/streptomycin (Gibco Life Technology). The isolation method of hADSCs was described in our previous study ${ }^{21}$. Three adipose tissue samples were obtained from patients (mean age: 21 years, range: 16-30 years) who underwent abdominal surgery or elective liposuction. The hADSCs were cultured in alpha minimum essential medium ( $\alpha$-MEM; Gibco Life Technology) supplemented with $10 \%$ FBS. All cells were cultured in a humidified $5 \% \mathrm{CO}_{2}$ atmosphere at $37^{\circ} \mathrm{C}$. When the cultures reached $\sim 80 \%$ confluence, the cells were detached by treatment with $0.05 \%$ trypsin/ethylenediaminetetraacetic acid (EDTA) and passaged, and the culture media were changed every 3 days.

\section{Induction of chondrogenesis in hADSCs}

The chondrogenesis induction method was described in our previous study ${ }^{6}$. In brief, cultured hADSCs were resuspended in incomplete chondrogenic medium $(194 \mathrm{~mL}$ human adipose mesenchymal stem cell chondrogenic differentiation basal medium, $20 \mu \mathrm{L}$ dexamethasone, $600 \mu \mathrm{L}$ ascorbate, $2 \mathrm{~mL}$ of ITS [insulin, transferrin, and selenium] supplement, $200 \mu \mathrm{L}$ sodium pyruvate, $200 \mu \mathrm{L}$ proline; Cyagen, Guangzhou, China), seeded in 24-well plates at a density of $10^{5}$ cells $/ \mu \mathrm{L}$ and incubated at $37^{\circ} \mathrm{C}$ for $70 \mathrm{~min}$. After incubation, $500 \mu \mathrm{l}$ of complete chondrogenic medium (incomplete chondrogenic medium with $5 \mu \mathrm{L}$ TGF- $\beta 3$ ) was added to each well, and the complete medium was changed every 3 days. Samples were collected at different time points for the experiments.

\section{RNA extraction, reverse transcription, and qRT-PCR}

The total RNA extraction was performed using the miRNeasy Mini Kit (QIAGEN, CA, USA), and cDNA was synthesized using the PrimeScript ${ }^{\circledR}$ miRNA cDNA Synthesis Kit (TaKaRa Bio, Japan). Quantitative real-time polymerase chain reaction (qRT-PCR) was performed using SYBR $^{\circledast}$ Premix Ex Taq ${ }^{\text {tw }}$ II (TaKaRa Bio, Japan) and a CFX96 real-time qPCR machine. The primers used for analysis are listed in Table 1 . Relative gene expression was 


\begin{tabular}{|c|c|c|}
\hline Gene & & Primer sequence $\left(5^{\prime}-3^{\prime}\right)$ \\
\hline has-GAPDH & $F$ & GCACCGTCAAGGCTGAGAAC \\
\hline has-GAPDH & $\mathrm{R}$ & ATGGTGGTGAAGACGCCAGT \\
\hline has-SOX9 & $\mathrm{F}$ & AGCGAACGCACATCAAGAC \\
\hline has-SOX9 & $\mathrm{R}$ & CTGTAGGCGATCTGTTGGGG \\
\hline has-COL2A1 & $\mathrm{F}$ & TGGACGATCAGGCGAAACC \\
\hline has-COL2A1 & $\mathrm{R}$ & GCTGCGGATGCTCTCAATCT \\
\hline has-ACAN & $\mathrm{F}$ & GTGCCTATCAGGACAAGGTCT \\
\hline has-ACAN & $\mathrm{R}$ & GATGCCTITCACCACGACTTC \\
\hline has-RUNX2 & $\mathrm{F}$ & CACTGGCGCTGCAACAAGA \\
\hline has-RUNX2 & $\mathrm{R}$ & CATTCCGGAGCTCAGCAGAATAA \\
\hline has-COL10A1 & $\mathrm{F}$ & CATAAAAGGCCCACTACCCAAC \\
\hline has-COL10A1 & $\mathrm{R}$ & ACCTTGCTCTCCTCTTACTGC \\
\hline has-MMP13 & $\mathrm{F}$ & TCCTGATGTGGGTGAATACAATG \\
\hline has-MMP13 & $\mathrm{R}$ & GCCATCGTGAAGTCTGGTAAAAT \\
\hline has-PAK2 & $\mathrm{F}$ & CAGAAACAGCCAAAGAAGGAAC \\
\hline has-PAK2 & $\mathrm{R}$ & AACGATGTTGGGATTITTCAA \\
\hline has-U6 & $\mathrm{F}$ & CTCGCTTCGGCAGCACA \\
\hline has-U6 & $\mathrm{R}$ & AACGCTTCACGAATTTGCGT \\
\hline has-455-3P & $F$ & GCAGTCCATGGGCATATACAC \\
\hline
\end{tabular}

calculated using the $2^{-\Delta \Delta t}$ method. All experiments were performed in triplicate.

\section{Transfection}

PHCs were transfected with a miR-455-3p mimic (RiboBio, Guangzhou, China) at a concentration of $50 \mathrm{nM}$ or an inhibitor of miR-455-3p at a concentration of 100 nM. A nonspecific miRNA (miR-Control; RiboBio) was used as a control. PHCs were also transfected with siPAK2 (50 nM) and siNC (RiboBio) as negative controls. Lipofectamine $^{\circledR} 2000$ Transfection Reagent (Gibco Life Technologies) was used to transfect PHCs according to the manufacturer's instructions.

\section{Western blot analysis}

The protein collection and western blot protocols were described in our previous study ${ }^{8}$. The nuclear proteins were isolated using a Nuclear Extraction kit (CW0199, CoWin Biosciences). Briefly, $20 \mu \mathrm{g}$ of protein was separated by sodium dodecyl sulfate-polyacrylamide gel electrophoresis (SDS-PAGE) and transferred to the polyvinylidene difluoride (PVDF) membranes (Millipore, Bedford USA). The membranes were incubated overnight at $4{ }^{\circ} \mathrm{C}$ with primary antibodies against PAK2 (1:1000 dilution, Cell Signaling Technology, \#2615), phosphoPAK2 (1:100 dilution, Abcam, ab40795), RUNX2 (1:1000 dilution, Abcam, ab76956), GAPDH (1:1000 dilution, CST), PCNA (1:1000 dilution, Proteintech, 10205-2-AP), COL2A1 (1:1000 dilution, Abcam, ab188570), MMP13 (1:1000 dilution, Abcam, ab39012), SOX9 (1:2000 dilution, Millipore, ABE2868), Smad2 (1:1000 dilution, CST, \#5339 S), Smad3 (1:1000 dilution, CST, \#9523), phosphoSmad2 (1:1000 dilution, CST, \#18338) and phosphoSmad3 (1:1000 dilution, CST, \#9520). After incubation with primary antibodies, the membranes were incubated with the corresponding horseradish peroxidase (HRP)conjugated secondary antibodies (1:3000 dilution, Cell Signaling Technology) at room temperature for $1 \mathrm{~h}$.

\section{Immunohistochemical analysis and in situ hybridization}

The methods for immunohistochemistry and in situ hybridization were described in our previous study ${ }^{20,22}$. For in situ hybridization, a probe for human miR-455-3p (Exiqon, Invitrogen, Shanghai, China) was used. For immunohistochemical analysis, deparaffinization and rehydration of the sections were performed with standard xylene-to-ethanol washes. The sections were then blocked in phosphate-buffered saline (PBS) plus $0.025 \%$ Tween 20 with $10 \%$ FBS. After blocking, the sections were incubated at $4{ }^{\circ} \mathrm{C}$ overnight with primary antibodies specific for PAK2 (1:100 dilution, Abcam, ab76293), phospho-PAK2 (1:100 dilution, Abcam, ab40795), COL2A1 (1:100 dilution, Abcam, ab188570), and MMP13 (1:80 dilution, Abcam, ab39012). Negative controls were prepared by substituting PBS for the primary antibodies. After overnight incubation, the sections were incubated with HRPconjugated anti-rabbit IgG secondary antibody (Cell Signaling Technology, Boston, USA) for $30 \mathrm{~min}$.

\section{Cell viability assay}

OA chondrocytes (2000 cells/well) were seeded into 96well plates. When the cultures reached $\sim 80 \%$ confluence, the supernatant was removed, and media with different concentrations of IPA-3 were added to the OA chondrocytes. At the indicated time points, the cell viability was measured using a Cell Counting Kit-8 (CCK-8, Dojindo Laboratories, Kumamoto, Japan) according to the manufacturer's instructions.

\section{Luciferase constructs and luciferase reporter assay}

PAK2 3'UTR DNA was amplified by PCR using the forward primer 5'-ATAGGCCGGCATAGACGCGTCA TCACTGCTGTGGCCTCATACTC-3' and the reverse primer 5'-AAAGATCCTTTATTAAGCTTGGGGAGGG GAGAAGGGAGG-3'. Seed sequences were mutated using PCR with the forward primer 5'-TAGTGA TCTTTCGTGCAATTCCTTCTGGACCCTAAAGAAG G-3' and the reverse primer 5'-AGAAGGAATTGCACG 
AAAGATCACTAGCCTTAGGTCTTTCAGCAAAC-3' . The amplified DNA sequences were inserted into the pmiR-RB-REPORT ${ }^{\mathrm{mm}}$ Vector (OBIO, Shanghai, China) to generate WT or mutant PAK2 3'UTR luciferase vectors. For the luciferase reporter assay, $1.2 \times 10^{4}$ cells (HEK293) were cotransfected with $100 \mathrm{nM} \mathrm{miR-455-3p}$ or miRControl and $0.2 \mu \mathrm{g}$ of vector containing WT or mutant PAK2 3'UTR in a 96-well plate. After $48 \mathrm{~h}$ of transfection, the Dual-Luciferase ${ }^{\circledast}$ Reporter Assay System (Promega Corp, Madison, WI, USA) was used to measure luciferase activity. Firefly luciferase activity was normalized to the Renilla luciferase activity. Luciferase assays were performed in quadruplicate and repeated in three independent experiments.

\section{Statistical analysis}

All experiments were performed with at least three biological replicates, and the data are presented as the mean \pm standard deviation (SD). Student's $t$ tests or Mann-Whitney U tests were used to identify differences between groups. The Gaussian distribution of the data was confirmed using the Shapiro-Wilk test. One-way analysis of variance (ANOVA) and Kruskal-Wallis tests were carried out for multiple group comparisons. $P$-values of $<0.05$ were considered statistically significant. All analyses were performed using SPSS software, version 13.0 (IBM Corporation, Armonk, NY, USA).

\section{Results}

Knee cartilage phenotype in miR-455-3p knockout mice

To investigate the role of miR-455-3p in cartilage development, we compared 5-month-old and 12-monthold miR-455-3p-deletion mice and wild-type mice. As shown in Fig. 1a, in situ hybridization of miR-455-3p confirmed the deletion efficiency in 12-month-old wildtype and mutant mice. MiR-455-3p was significantly decreased in $\mathrm{KO}$ mouse cartilage. In addition, the miR455-3p KO mice showed surface irregularities on the articular surfaces of the knee joint, increased hypocellularity, and decreased Safranin O staining of the extracellular matrix compared with the WT mice. The Mankin score of the miR-455-3p mice was significantly higher than that of the WT mice at both 5 and 12 months of age (Fig. 1b-d). COL2A1 levels were significantly reduced in the miR-455-3p-deletion mice at 5 months and 12 months, while MMP13 expression was substantially increased in the mutant mice (Fig. 1b, c, e, f). These results indicated that miR-455-3p plays a crucial role in cartilage development and degeneration.

\section{Expression patterns of miR-455-3p and PAK2 during chondrogenesis of hADSCs}

To investigate the regulatory mechanism of miR-455-3p in chondrocytes and confirm our hypothesis, we studied this mechanism in vitro. Chondrogenesis of hADSCs was induced by TGF- $\beta 3$. As shown in Fig. $2 a$, miR- $455-3 p$ expression increased rapidly at the beginning of day 3 , peaked at day 21, and then sharply decreased from day 28 to day 35. However, an opposite expression pattern was observed for PAK2 and miR-455-3p during chondrogenic differentiation from days 14 to 35 (Fig. 2b-e), indicating that the expression levels of miR-455-3p and PAK2 may be related to cartilage degeneration and that the expression of PAK2 may be affected by miR-455-3p. Finally, chondrogenesis of hADSCs was confirmed by the expression levels of COL2A1, which increased during early chondrogenesis and decreased in late-stage chondrogenesis (Fig. 2c).

\section{Expression levels of miR-455-3p and PAK2 in human OA and control cartilage}

To further determine whether the expression levels of miR-455-3p and PAK2 changed during cartilage degeneration, we compared the expression levels of miR-455-3p and PAK2 in human control and OA cartilage by qRTPCR and western blotting. The miR-455-3p levels were decreased, and both PAK2 and phospho-PAK2 were increased in OA cartilage compared with control cartilage (Fig. 3a-d). This trend was confirmed by in situ hybridization (Fig. 3e-g) and immunohistochemical analysis (Fig. $3 \mathrm{~h}-\mathrm{j}$ ). These results further indicated that miR-455-3p and PAK2 are involved in OA progression and that PAK2 may be regulated by miR-455-3p.

\section{PAK2 knockdown has effects similar to those of miR-455- $3 p$ overexpression on $O A$ chondrocytes}

To further investigate the role of miR-455-3p and PAK2 in OA cartilage, we transfected OA chondrocytes with miR-455-3p, anti-miR-455-3p, and/or siPAK2. After transfection, the expression levels of cartilage-specific genes (SOX9, COL2A1, and ACAN) and hypertrophyrelated genes (RUNX2, COL10A1, and MMP13) were determined by qRT-PCR, and the protein levels of SOX9, COL2A1, RUNX2, and MMP13 were assessed by western blotting. Overexpression of miR-455-3p in OA chondrocytes promoted the expression of cartilage-specific genes and decreased the expression of hypertrophyrelated genes. In contrast, an opposing expression pattern of these genes was observed in OA chondrocytes with miR-455-3p silencing (Fig. 4a-d). Similar relationships between miR-455-3p and the expression levels of SOX9, COL2A1, RUNX2, and MMP13 were detected by western blotting (Fig. 4e-f). The knockdown of PAK2 was correlated with increased expression of cartilage-specific genes, whereas the expression of RUNX2, COL10A1, and MMP13 was downregulated (Fig. 4g-i). These results indicated that the level of miR-455-3p or PAK2 can regulate cartilage degeneration. 


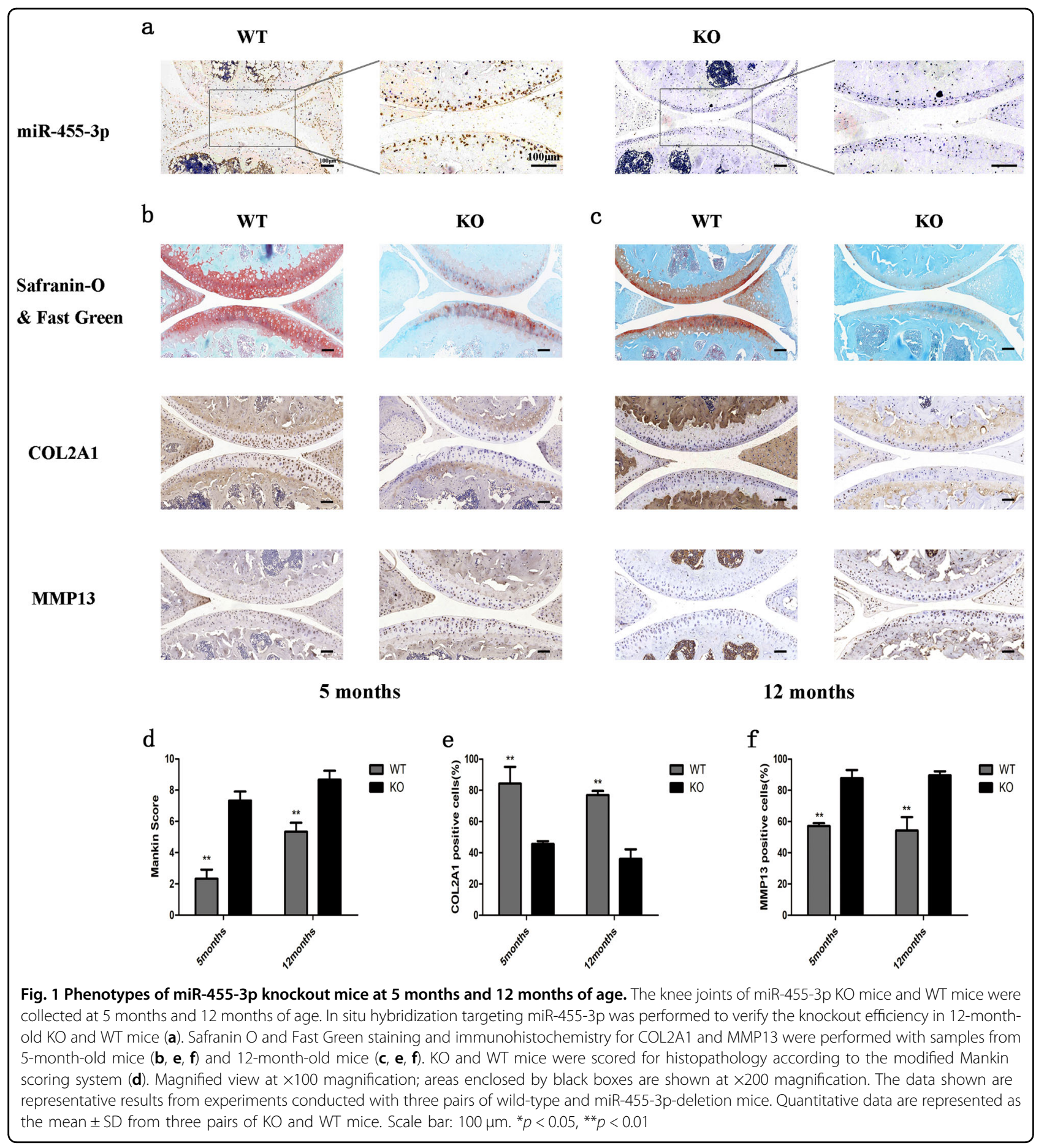

\section{MiR-455-3p suppresses PAK2 expression by directly targeting the PAK2 $3^{\prime}$ UTR}

Because of the similar effects of siPAK2 and the miR455-3p mimic, we hypothesized that miR-455-3p may regulate the degeneration of cartilage through downregulation of PAK2. PAK2 mRNA and protein levels were decreased in $\mathrm{OA}$ chondrocytes overexpressing
miR-455-3p and were increased upon addition of antimiR-455-3p (Fig. 5a-c). To investigate the molecular mechanisms underlying the regulation of PAK2 expression by miR-455-3p, we analyzed the 3'UTR of human PAK2 mRNA. Web servers that predicts biological targets of miRNAs, such as TargetScan (http://www. targetscan.org) and miRanda (http://www.microrna. 


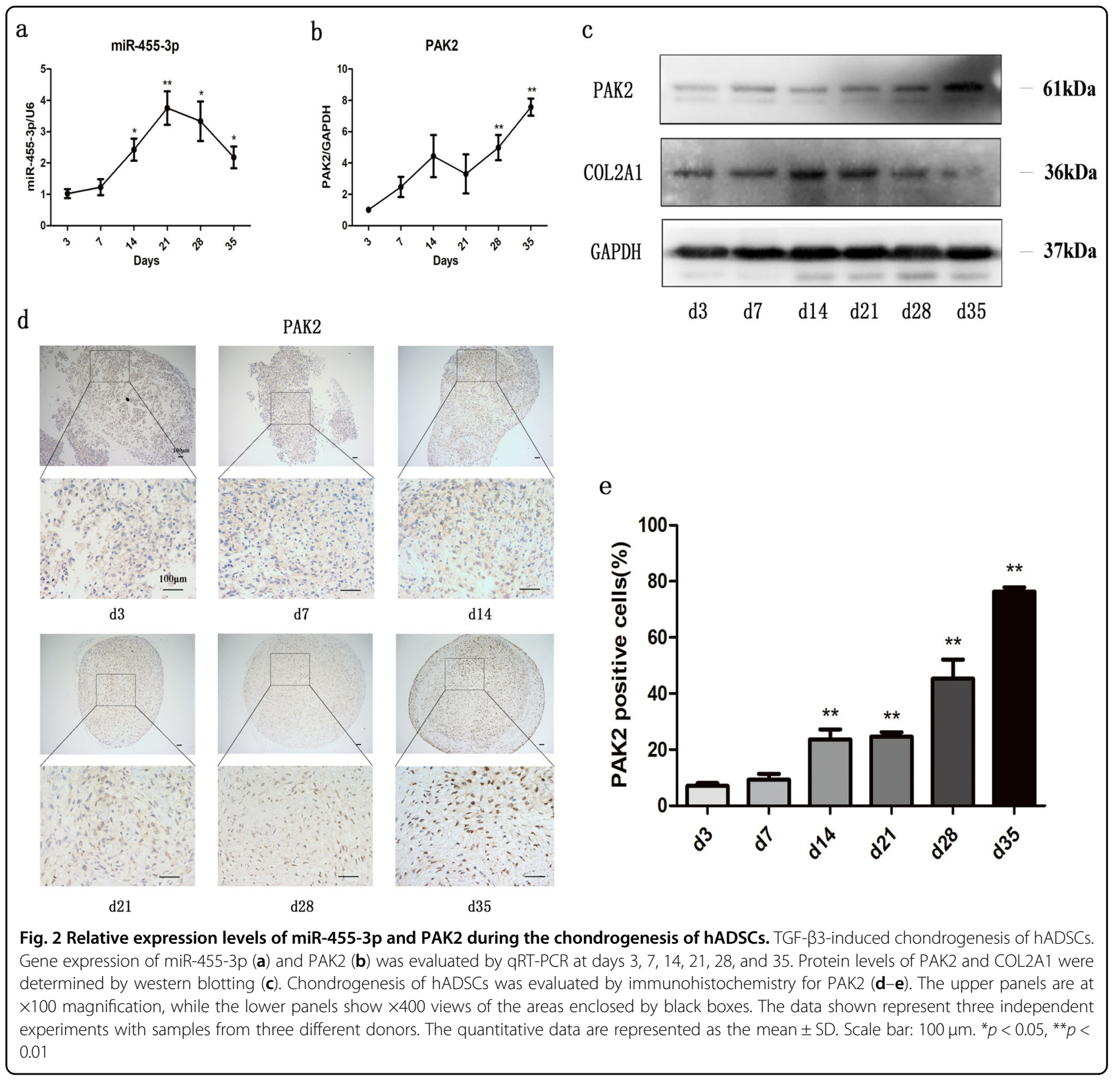

org), revealed that the $3^{\prime} \mathrm{UTR}$ of human PAK2 contains a potential binding site for miR-455-3p (Fig. 5d). We therefore utilized a luciferase reporter assay to test this potential interaction. Cotransfection of miR-455-3p and PAK2 3'UTR luciferase reporter plasmids significantly reduced the luciferase activity, whereas a mutated PAK2 3'UTR sequence prevented this reduction (Fig. 5e). We further confirmed this regulatory relationship between miR-455-3p and PAK2 in vivo. The expression of PAK2 (Fig. 5f, g) and phospho-PAK2 (Fig. 5h, i) was significantly increased in miR-455-3p KO mice compared with WT mice at 12 months of age. These results confirmed that miR-455-3p regulates PAK2 expression by binding the PAK2 3'UTR.
PAK2 knockdown blocks the effects of the miR-455-3p inhibitor on $\mathrm{OA}$ chondrocytes

To confirm that miR-455-3p regulates chondrocyte metabolism by targeting PAK2, we cotransfected OA chondrocytes with siPAK2 and anti-miR-455-3p to examine whether the effects of the miR-455-3p inhibitor could be blocked by PAK2 knockdown. We found that the miR-455-3p inhibitor decreased the expression of cartilage-specific genes and increased the expression of hypertrophy-related genes and that PAK2 siRNA significantly blocked these effects (Fig. 5 j-p). Similar results were observed with western blotting (Fig. 5q). These results indicated that $\mathrm{miR}-455-3 \mathrm{p}$ regulates OA chondrocytes through targeting PAK2. 


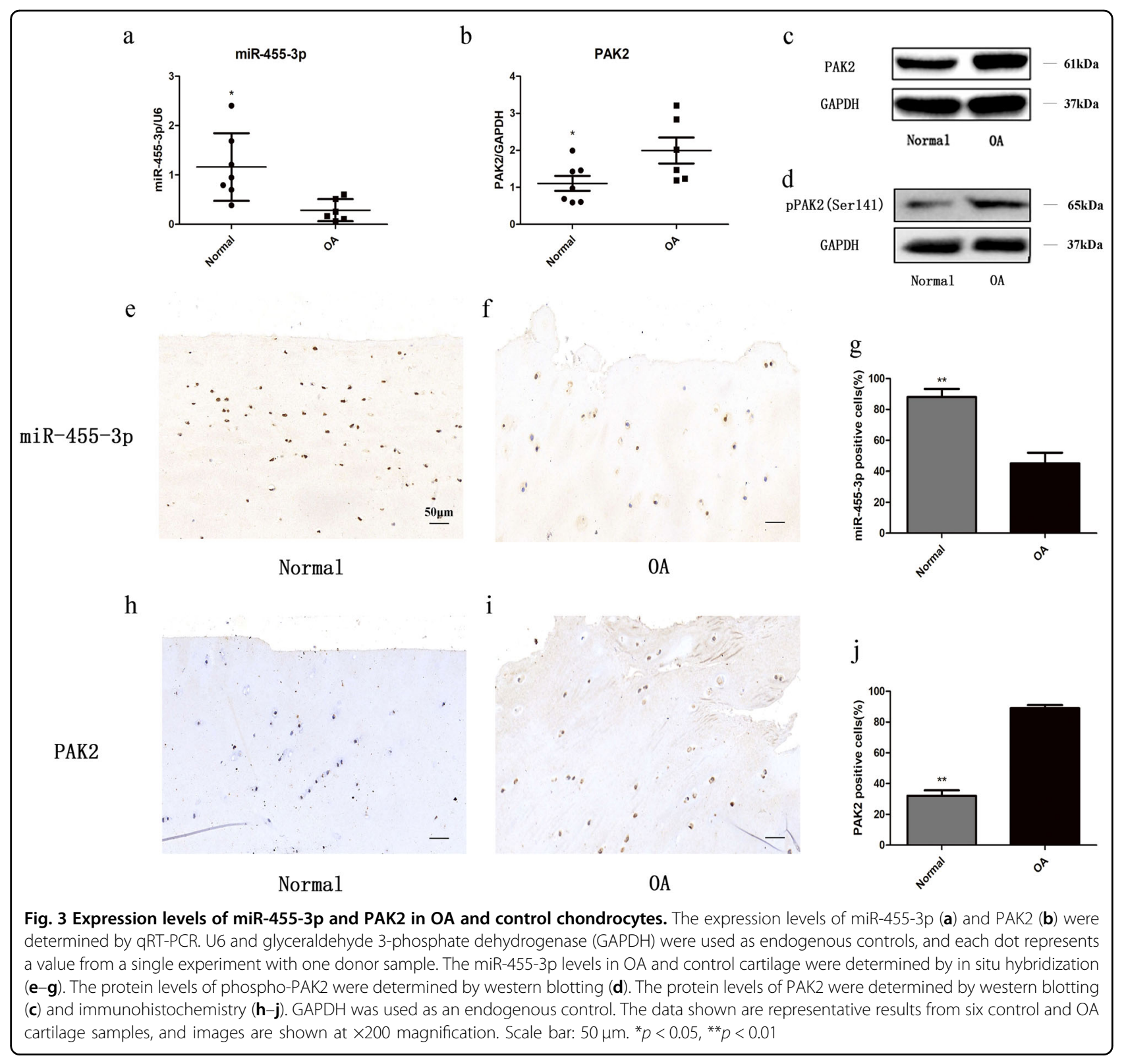

\section{Effects of IPA-3, a PAK inhibitor, on OA chondrocytes}

To further assess the role of PAK2 in cartilage, we treated OA chondrocytes with IPA-3, an allosteric PAK inhibitor $^{23}$. Since $10 \mu \mathrm{M}$ IPA-3 had no effect on the viability of OA chondrocytes after 3 days of treatment (Fig. $6 a)$, this concentration was utilized for subsequent experiments. First, PAK2 phosphorylation levels were reduced by IPA-3 treatment (Fig. 6b). Furthermore, $10 \mu \mathrm{M}$ IPA-3 increased the mRNA levels of SOX9, COL2A1, and ACAN, and decreased those of RUNX2, COL10A1, and MMP13 in a time-dependent manner (Fig. $6 \mathrm{c}-\mathrm{h})$. Similar trends were observed with western blotting (Fig. 6i). IPA-3 was dissolved in dimethyl sulfoxide (DMSO), and we used the same volume of DMSO for $3 \mathrm{~h}$ as a control; DMSO likely has no additional effects during the IPA-3 treatment (Fig. 6j, k). We further confirmed this finding through a time-course experiment in chondrocytes treated with DMSO (Supplementary Fig. 1). These results thus demonstrate that inhibiting PAK2 has positive effects on OA chondrocytes.

\section{IPA-3 and PAK2 knockdown promote TGF- $\beta$-induced R- Smad activation and signaling in chondrocytes}

To investigate whether PAK2 can affect the TGF- $\beta$ / Smad signaling pathway in chondrocytes, we transfected siPAK2 and siControl into PHCs. After $72 \mathrm{~h}$ of transfection, PAK2 knockdown increased the total and nuclear levels of phosphorylated Smad2 and Smad3 after $1 \mathrm{~h}$ of 


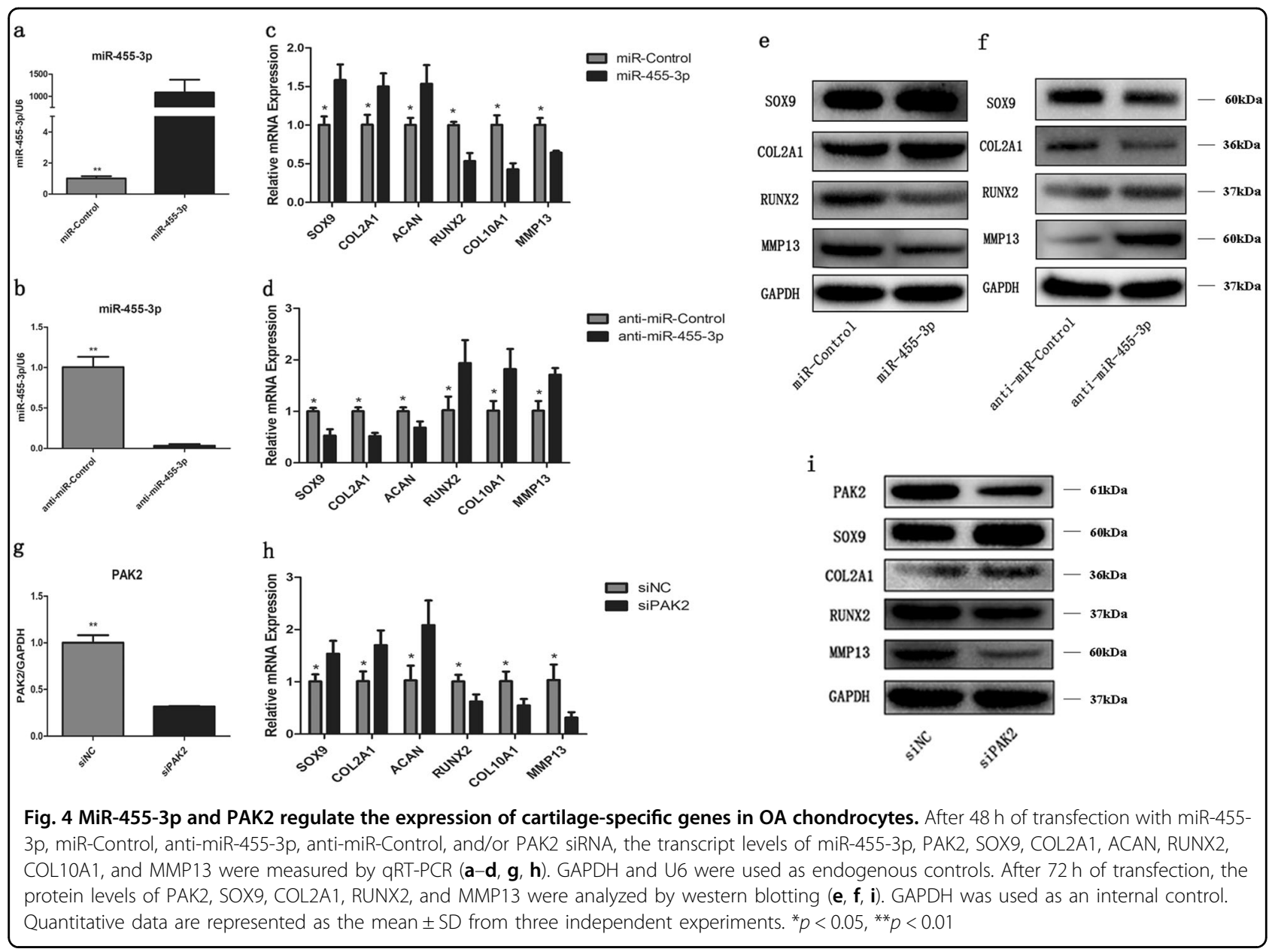

incubation with TGF- $\beta 1$ (5 ng/ml) (Fig. 7a, b, f). A similar trend was found in the PHCs treated with IPA-3 $(10 \mu \mathrm{M})$ compared with control cells (Fig. 7c, d, e). These results indicated that PAK2 is an antagonist of the TGF- $\beta / \mathrm{Smad}$ signaling pathway in chondrocytes.

\section{Discussion}

MiR-455-3p has attracted considerable attention in recent years due to its regulatory functions in most tissues and organs of the human body ${ }^{24,25}$. In our previous study, we demonstrated that miR-455-3p regulates chondrogenesis in $\mathrm{ATDC} 5^{8}$. However, the regulatory function of miR-455-3p in human OA chondrocytes is still unknown. In this study, we showed that miR-455-3p promotes TGF- $\beta$ signaling and inhibits cartilage degeneration in human chondrocytes by directly targeting PAK2.

The TGF- $\beta /$ Smad signaling pathway has been shown to play a key role in maintaining articular cartilage ${ }^{26}$. The levels of SOX9, COL2A1, and ACAN increased and those of RUNX2, COL10A1, and MMP13 decreased after activation of the TGF- $\beta /$ Smad signaling pathway in chondrocytes $^{27-30}$. Inhibiting the TGF- $\beta /$ Smad signaling pathway in chondrocytes led to hypertrophic differentiation of chondrocytes and progressive development of $\mathrm{OA}^{31,32}$. Our results using miR-455-3p knockout mice showed that both miR-455-3p and the TGF- $\beta /$ Smad signaling pathway are likely involved in cartilage development. As reported in a previous study on PAK2-mediated inhibition of the TGF- $\beta$ signaling pathway ${ }^{17}$, PAK2 phosphorylates Smad2 at Ser417 and Smad3 at Ser375, thus abolishing TGF- $\beta$-induced $\operatorname{Smad} 2 / 3$ activation and downstream signaling. Based on the bioinformatics predictions of miR-455-3p and PAK2 interactions, we hypothesized that miR-455-3p could promote TGF- $\beta$ / Smad signaling and inhibit cartilage degeneration by directly targeting PAK2.

We tested our hypothesis and found opposite expression patterns of miR-455-3p and PAK2 in the late stage of chondrogenesis of hADSCs. Contrasting expression trends of miR-455-3p and PAK2 were also observed in $\mathrm{OA}$ and control chondrocytes. These results indicated that decreased miR-455-3p levels and increased PAK2 levels may drive OA development. We then confirmed the 


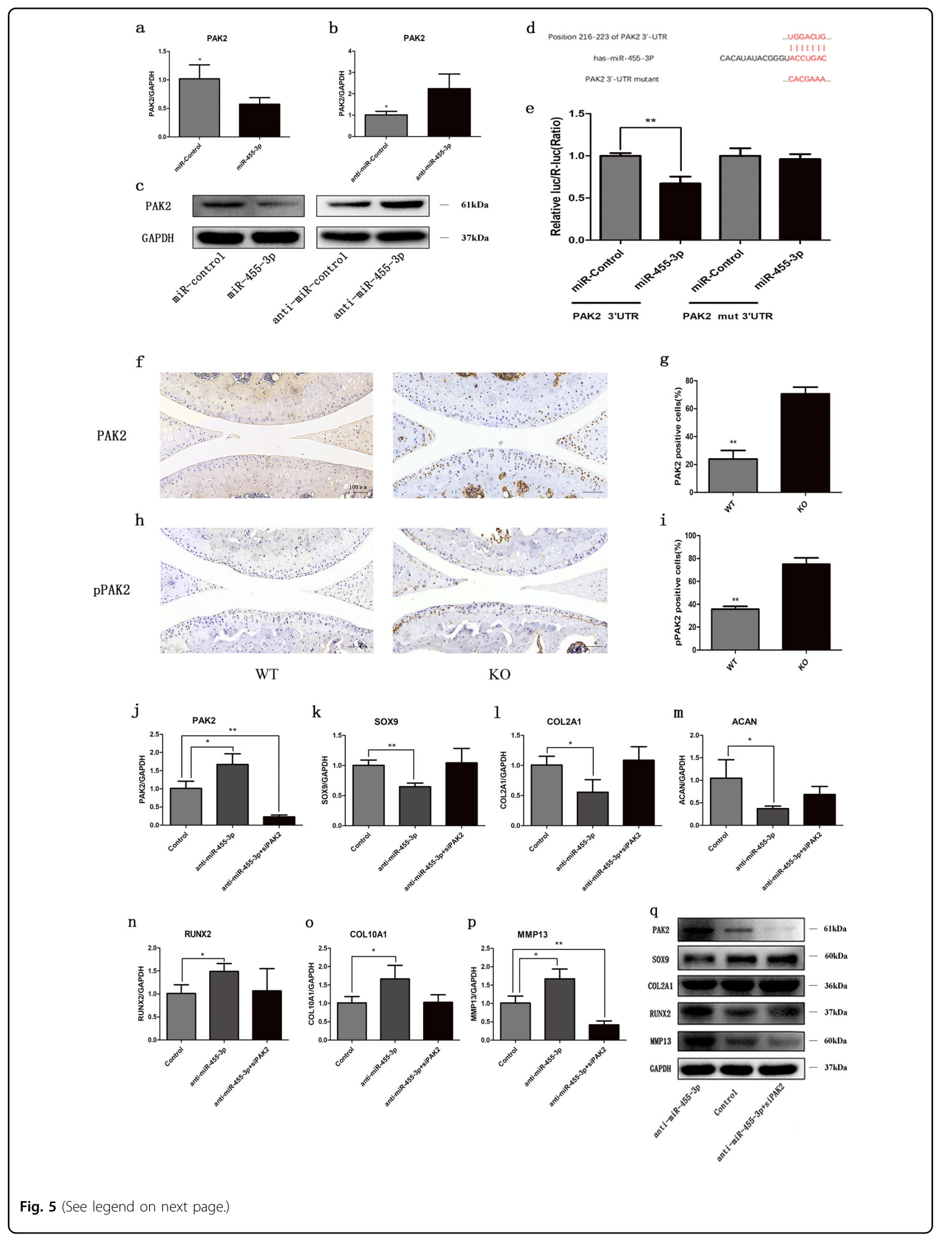


(see figure on previous page)

Fig. 5 MiR-455-3p regulates the expression of cartilage-specific genes by targeting PAK2. OA chondrocytes were transfected with miR-455-3p, miR-Control, anti-miR-455-3p, anti-miR-Control, or anti-miR-455-3p + siPAK2. The transcript levels of PAK2, SOX9, COL2A1, ACAN, RUNX2, COL10A1, and MMP13 were measured by qRT-PCR after $48 \mathrm{~h}$ of transfection $(\mathbf{a}, \mathbf{b}, \mathbf{j}-\mathbf{p})$. The protein levels of PAK2, SOX9, COL2A1, RUNX2, and MMP13 were visualized by western blotting after $72 \mathrm{~h}$ of transfection $(\mathbf{c}, \mathbf{q})$. The sequence alignment of miR-455-3p and PAK2 $3^{\prime} U T R$ is shown (d). First, 293T cells were cotransfected with either a WT or mutant PAK2 $3^{\prime} U$ TR reporter plasmid and either a miR-455-3p or anti-miR-455-3p construct. Cells were harvested for luciferase assays after $48 \mathrm{~h}$ of transfection $(\mathbf{e})$. Immunohistochemistry analysis of PAK2 (f- $-\mathbf{g})$ and phospho-PAK2 (h-i) was performed in 12-month-old wild-type and miR-455-3p knockout mice. Magnified view at $\times 150$ magnification. Quantitative data are represented as the mean \pm SD from three independent experiments. GAPDH was used as an internal control. Scale bar: $100 \mu \mathrm{m} .{ }^{*} p<0.05,{ }^{* *} p<0.01$

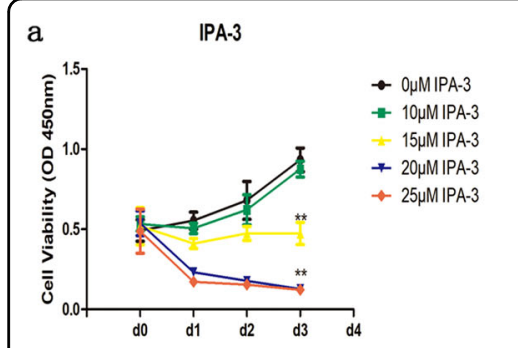

e

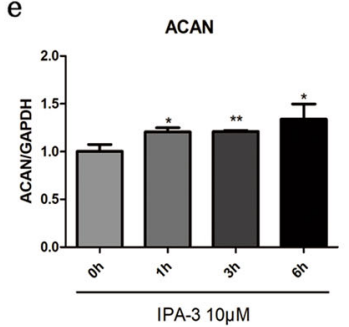

i
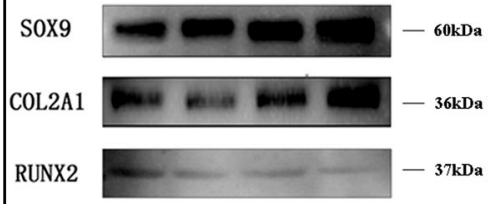

MMP13

GAPDH

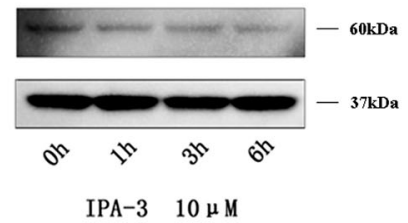

b

IPA-3 $(10 \mu$ N

pPAK2 (Ser141)

GAPDH

f

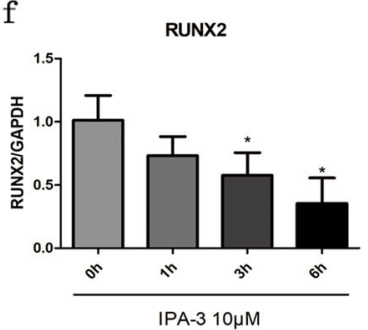

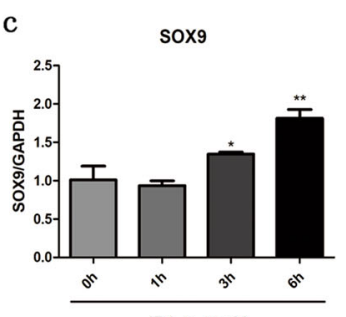

IPA-3 104M

g

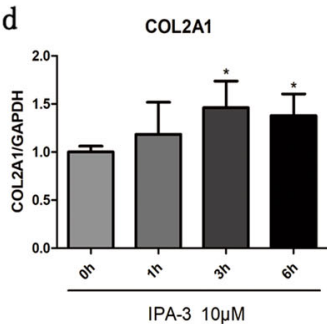

h

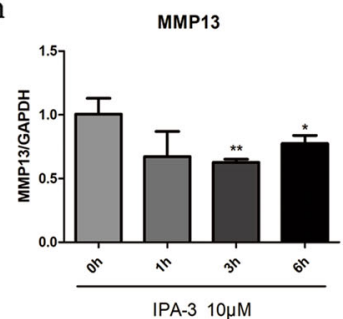

j

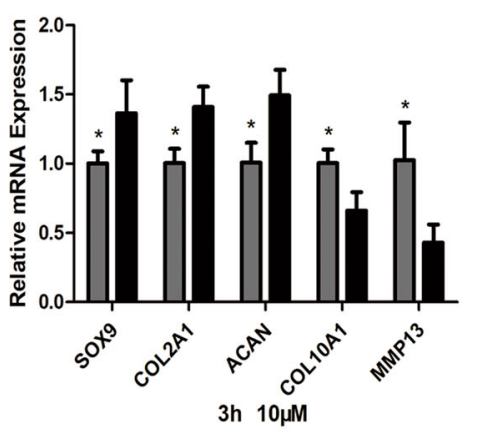

$\mathrm{k}$

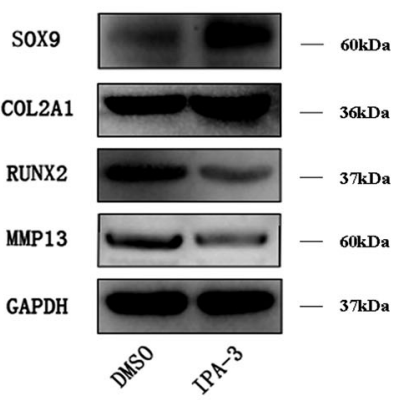

3h $10 \mu \mathrm{M}$

Fig. 6 IPA-3 regulates the expression of cartilage-specific genes in OA chondrocytes. OA chondrocytes were incubated with different concentrations of IPA-3. Cell viability was detected with CCK-8 assays at $d 0-d 3$ (a). OA chondrocytes were incubated with $10 \mu \mathrm{M}$ IPA-3, and the level of phospho-PAK2 was detected by western blotting after $1 \mathrm{~h}$ and $2 \mathrm{~h}$ of treatment $(\mathbf{b})$. The transcript levels of SOX9, COL2A1, ACAN, RUNX2, COL10A1, and MMP13 were measured by qRT-PCR at $0 \mathrm{~h}, 1 \mathrm{~h}, 3 \mathrm{~h}$, and $6 \mathrm{~h}$ of IPA-3 treatment (c-i). The protein levels of SOX9, COL2A1, RUNX2, and MMP13 were visualized using western blotting (i). OA chondrocytes were incubated with $10 \mu \mathrm{M} \mathrm{IPA-3}$ or DMSO, and the levels of transcripts and proteins were measured after $3 \mathrm{~h}$ of incubation $(\mathbf{j}-\mathbf{k})$. Quantitative data are represented as the mean $\pm \mathrm{SD}$ from three independent experiments. GAPDH was used as an internal control. ${ }^{*} p<0.05,{ }^{* *} p<0.01$

effects of miR-455-3p overexpression or PAK2 knockdown on OA chondrocytes and observed increased SOX9, COL2A1, and ACAN expression, and decreased RUNX2, COL10A1, and MMP13 expression. These effects were similar to those observed upon activation of the TGF- $\beta$ /
Smad signaling pathway in chondrocytes. Moreover, these results further confirmed the importance of miR-455-3p and PAK2 in OA development. In addition, due to the opposite expression patterns and similar effects of these molecules, we proposed that miR-455-3p can regulate the 


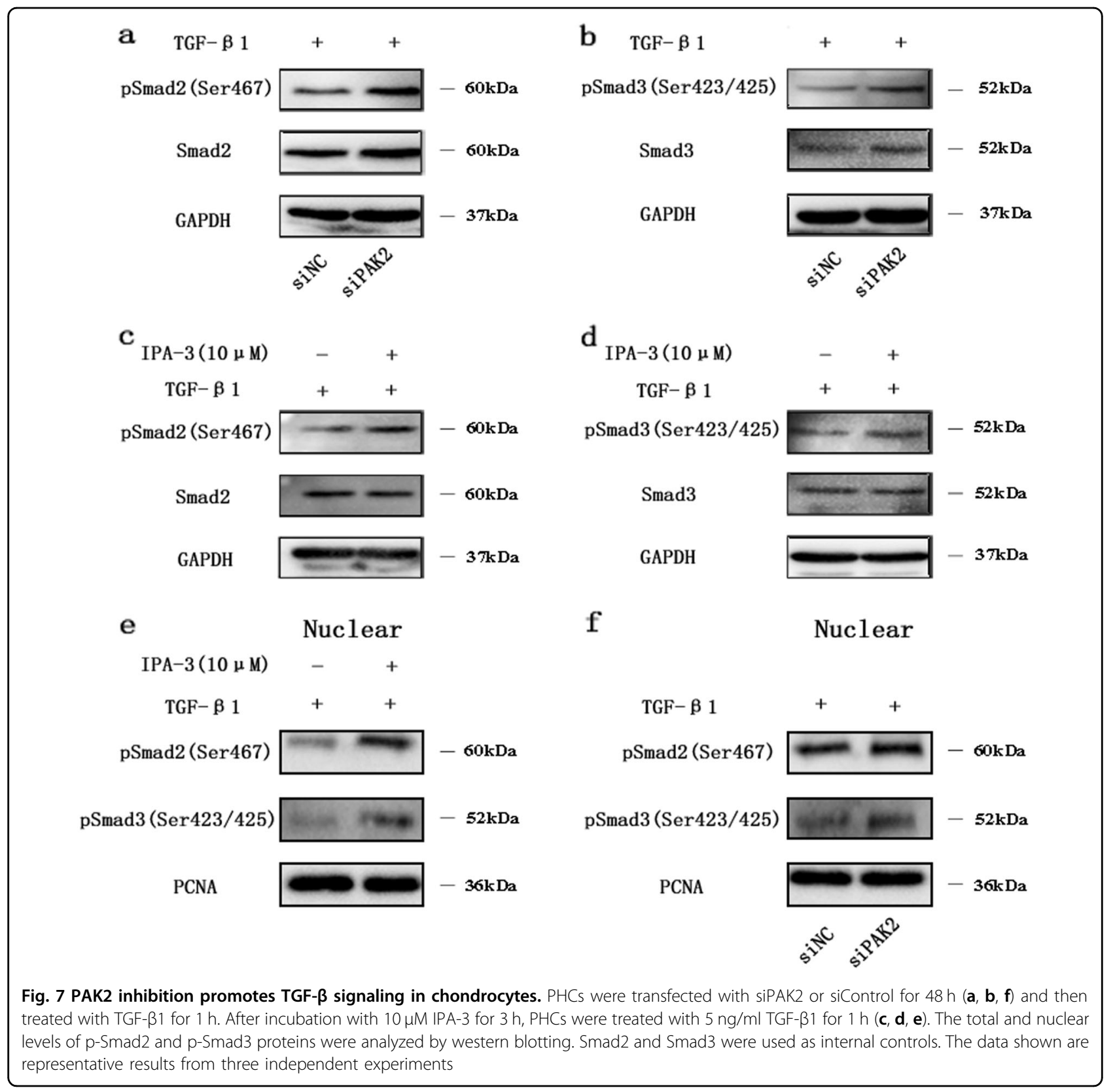

expression of PAK2 by directly targeting its 3'UTR sequence. A luciferase reporter assay was performed to clarify the underlying regulatory mechanism. In addition, anti-miR-455-3p and siPAK2 were cotransfected into OA chondrocytes to confirm that the regulatory functions of miR-455-3p in OA chondrocytes are mediated by targeting PAK2. We also used IPA-3, a PAK inhibitor, to further clarify the role of PAK2 in OA chondrocytes. Finally, we determined the levels of p-Smad2 and pSmad3 to demonstrate that inhibiting PAK2 can promote TGF- $\beta /$ Smad signaling in chondrocytes. Based on our results, we concluded that miR-455-3p inhibits cartilage degeneration via suppressing PAK2 expression and promoting the activity of the TGF- $\beta /$ Smad signaling pathway. A previous study ${ }^{33}$ demonstrated that miR-4553 p suppresses the $S \operatorname{mad} 2 / 3$ pathway, as shown by luciferase assays. However, these experiments were performed in SW-1353 chondrosarcoma cells, not in chondrocytes, which may be the reason for the discrepancy between the results of the two studies.

This study has several limitations. First, the reasons for miR-455-3p and PAK2 overexpression in the early stages of hADSC chondrogenesis were not clarified. Second, IPA-3 is not a PAK2-specific inhibitor. Although it would have been more appropriate to use a PAK2-specific inhibitor, IPA3 has also been widely used in previous 
PAK2 studies ${ }^{17,34}$. In addition, while our findings demonstrated that miR-455-3p may be an important factor in $\mathrm{OA}$ development through $\mathrm{KO}$ mice, future investigation of the destabilization of the medial meniscus model (DMM) in miR-455-3p KO mice is needed to further characterize the role of miR-455-3p in OA progression. This study also demonstrated that miR-455-3p regulates the TGF- $\beta$ signaling pathway by targeting PAK2; however, further studies will be needed to provide more direct evidence that miR-455-3p is an upstream regulator of the TGF- $\beta$ signaling pathway. Finally, to our knowledge, there has been little research conducted on PAK2 in OA. We performed primary research on the role of PAK2 in OA chondrocytes. The regulatory mechanism of PAK2 in OA requires further study.

Altogether, our results indicate that miR-455-3p can promote the TGF- $\beta /$ Smad signaling pathway in chondrocytes and inhibit cartilage degeneration by directly suppressing PAK2. Thus, miR-455-3p may serve as a novel therapeutic agent and PAK2 a therapeutic target in OA.

\section{Acknowledgements}

This study was funded by the National Nature Science Foundation of China (81874016, 81672145, 81472101, 81572119, 81874014, 81672198, 81301558, 81601921, and 81702141), the Science and Technology Planning Project of Guangdong Province, China (2016A020214005, 2015A020212015, and 2019A030317007), and the Science and Technology Project of Guangzhou City, China (201710010164). We thank Zhiyu Huang and Guangxin Huang for their help in techniques for animal experimentation, and thank Anyu Zeng and Ruifu Lin for their help in the isolation of primary human chondrocytes.

\section{Conflict of interest}

The authors declare that they have no conflict of interest.

\section{Publisher's note}

Springer Nature remains neutral with regard to jurisdictional claims in published maps and institutional affiliations.

Supplementary information accompanies this paper at https://doi.org/ 10.1038/s12276-019-0322-3.

Received: 1 March 2019 Revised: 13 August 2019 Accepted: 22 August 2019.

Published online: 4 October 2019

\section{References}

1. Lawrence, R. C. et al. Estimates of the prevalence of arthritis and other rheumatic conditions in the United States. Part II. Arthritis Rheum. 58, 26-35 (2008).

2. Kapoor, M., Martel-Pelletier, J., Lajeunesse, D., Pelletier, J. P. \& Fahmi, H. Role of proinflammatory cytokines in the pathophysiology of osteoarthritis. Nat. Rev. Rheumatol. 7, 33-42 (2011).

3. Engels, B. M. \& Hutvagner, G. Principles and effects of microRNA-mediated post-transcriptional gene regulation. Oncogene 25, 6163-6169 (2006).

4. Zhang, Y. et al. MicroRNA-21 controls the development of osteoarthritis by targeting GDF-5 in chondrocytes. Exp. Mol. Med. 46, e79 (2014).

5. Makki, M. S. \& Haqqi, T. M. miR-139 modulates MCPIP1/L-6 expression and induces apoptosis in human OA chondrocytes. Exp. Mol. Med. 47, e189 (2015).

6. Zhang, Z. et al. Expression of microRNAs during chondrogenesis of human adipose-derived stem cells. Osteoarthr. Cartil. 20, 1638-1646 (2012).
7. Zhang, Z. et al. MiR-455-3p regulates early chondrogenic differentiation via inhibiting Runx2. FEBS Lett. 589, 3671-3678 (2015).

8. Chen, W. et al. MicroRNA-455-3p modulates cartilage development and degeneration through modification of histone $\mathrm{H} 3$ acetylation. Biochimica et. Biophysica Acta (BBA) - Mol. Cell Res. 1863, 2881-2891 (2016).

9. Tchetina, E. V., Antoniou, J., Tanzer, M., Zukor, D. J. \& Poole, A. R. Transforming growth factor-beta2 suppresses collagen cleavage in cultured human osteoarthritic cartilage, reduces expression of genes associated with chondrocyte hypertrophy and degradation, and increases prostaglandin E(2) production. Am. J. Pathol. 168, 131-140 (2006).

10. Ulrich-Vinther, M., Stengaard, C., Schwarz, E. M., Goldring, M. B. \& Soballe, K. Adeno-associated vector mediated gene transfer of transforming growth factor-beta1 to normal and osteoarthritic human chondrocytes stimulates cartilage anabolism. Eur. Cell Mater. 10, 40-50 (2005).

11. Taglieri, D. M. et al. Ablation of p21-activated kinase-1 in mice promotes isoproterenol-induced cardiac hypertrophy in association with activation of Erk1/2 and inhibition of protein phosphatase 2A. J. Mol. Cell Cardiol. 51, 988-996 (2011).

12. Knaus, U. G., Wang, Y., Reilly, A. M., Warnock, D. \& Jackson, J. H. Structural requirements for PAK activation by Rac GTPases. J. Biol. Chem. 273, 21512-21518 (1998).

13. Jaffer, Z. M. \& Chernoff, J. p21-Activated kinases: three more join the Pak. Int. J. Biochem. Cell Biol. 34, 713-717 (2002).

14. Dummler, B., Ohshiro, K., Kumar, R. \& Field, J. Pak protein kinases and their role in cancer. Cancer Metastasis Rev. 28, 51-63 (2009).

15. Li, T. et al. P21-activated protein kinase (PAK2)-mediated c-Jun phosphorylation at 5 threonine sites promotes cell transformation. Carcinogenesis $\mathbf{3 2}$, 659-666 (2011).

16. Wu, R., Abramson, A. L., Symons, M. H. \& Steinberg, B. M. Pak1 and Pak2 are activated in recurrent respiratory papillomas, contributing to one pathway of Rac1-mediated COX-2 expression. Int J. Cancer 127, 2230-2237 (2010).

17. Yan, X. et al. p21-activated kinase 2 (PAK2) inhibits TGF- $\beta$ signaling in MadinDarby canine kidney (MDCK) epithelial cells by interfering with the receptorsmad interaction. J. Biol. Chem. 287, 13705-13712 (2012).

18. Sun, $\mathrm{H}$. et al. MiR-455-3p inhibits the degenerate process of chondrogenic differentiation through modification of DNA methylation. Cell Death Dis. 9, 537 (2018).

19. Thomas, M. et al. Losartan attenuates progression of osteoarthritis in the synovial temporomandibular and knee joints of a chondrodysplasia mouse model through inhibition of TGF- $\beta 1$ signaling pathway. Osteoarthr. Cartil. 27, 676-686 (2019).

20. Hou, C. et al. The role of MicroRNA-381 in chondrogenesis and interleukin-1- $\beta$ induced chondrocyte responses. Cell Physiol. Biochem 36, 1753-1766 (2015).

21. Zhang, Z. et al. miRNA expression profile during osteogenic differentiation of human adipose-derived stem cells. J. Cell Biochem 113, 888-898 (2012).

22. Meng, F. et al. MicroRNA-320 regulates matrix metalloproteinase-13 expression in chondrogenesis and interleukin-1beta-induced chondrocyte responses. Osteoarthr. Cartil. 24, 932-941 (2016).

23. Deacon, S. W. et al. An isoform-selective, small-molecule inhibitor targets the autoregulatory mechanism of p21-activated kinase. Chem. Biol. 15, 322-331 (2008).

24. Kumar, S., Vijayan, M. \& Reddy, P. H. MicroRNA-455-3p as a potential peripheral biomarker for Alzheimer's disease. Hum. Mol. Genet 26, 3808-3822 (2017).

25. Wu, J. et al. MiR-455-3p suppresses renal fibrosis through repression of ROCK2 expression in diabetic nephropathy. Biochem Bioph Res Co. 503, 977-983 (2018).

26. Shen, J., Li, S. \& Chen, D. TGF- $\beta$ signaling and the development of osteoarthritis. Bone Res. 214002 (2014).

27. Chavez, R. D., Coricor, G., Perez, J., Seo, H. S. \& Serra, R. SOX9 protein is stabilized by TGF- $\beta$ and regulates PAPSS 2 mRNA expression in chondrocytes. Osteoarthr. Cartil. 25, 332-340 (2017).

28. Zhu, Y. et al. Transforming growth factor-betal induces type II collagen and aggrecan expression via activation of extracellular signal-regulated kinase $1 / 2$ and Smad2/3 signaling pathways. Mol. Med Rep. 12, 5573-5579 (2015).

29. Kang, J. S., Alliston, T., Delston, R. \& Derynck, R. Repression of Runx2 function by TGF-beta through recruitment of class II histone deacetylases by Smad3. Embo J. 24, 2543-2555 (2005).

30. Chen, C. G., Thuillier, D., Chin, E. N. \& Alliston, T. Chondrocyte-intrinsic Smad3 represses Runx2-inducible matrix metalloproteinase 13 expression to maintain articular cartilage and prevent osteoarthritis. Arthritis Rheumatism 64, 3278-3289 (2012). 
31. Shen, J. et al. Deletion of the transforming growth factor $\beta$ receptor type ॥ gene in articular chondrocytes leads to a progressive osteoarthritis-like phenotype in mice. Arthritis Rheumatism 65, 3107-3119 (2013).

32. Yang, X. et al. TGF- $\beta / S$ mad3 signals repress chondrocyte hypertrophic differentiation and are required for maintaining articular cartilage. J. Cell Biol. 153, 35-46 (2001)
33. Swingler, T. E. et al. The expression and function of microRNAs in chondrogenesis and osteoarthritis. Arthritis Rheumatism 64, 1909-1919 (2012).

34. Nuche-Berenguer, B., Ramos-Álvarez, I. \& Jensen, R. T. The p21-activated kinase PAK2, is important in the activation of numerous pancreatic acinar cell signaling cascades and in the onset of early pancreatitis events. Biochimica et. Biophysica Acta (BBA) - Mol. Basis Dis. 1862, 1122-1136 (2016). 